\title{
Dermatomiositis y colitis ulcerosa: presentación de un caso
}

\section{A Case of Dermatomyositis and Ulcerative Colitis}

\author{
Pedro Rosales Torres, ${ }^{1 *}$ Rafael Pila Pérez, ${ }^{1}$ Rafael Pila Peláez, ${ }^{1}$ Javier Artola González. ${ }^{1}$
}

1 Hospital Provincial Docente Manuel Ascunce Domenech. Camagüey, Cuba.

*Correspondencia: rtpedro.cmw@infomed.sld.cu.

Fecha recibido: $\quad 22 / 02 / 18$

Fecha aceptado: $30 / 04 / 18$

\begin{abstract}
Resumen
Introducción: la incidencia de la miopatía inflamatoria idiopática es de 4 a 15 casos por millón de habitantes y su prevalencia es de 60 casos por cada millón de habitantes. Dada la identificación de formas clínicas es posible que estas cifras de incidencia y prevalencia aumenten en los próximos años. La colitis ulcerosa ha ido en aumento afectando al $2 \%$ de la población mundial. Los síntomas están determinados por la extensión y gravedad de las lesiones, teniendo un curso crónico que puede evolucionar por brotes y no existe tratamiento médico curativo. La asociación de la dermatomiositis y la colitis ulcerativa es extremadamente infrecuente. Objetivo: Presentar un caso diagnosticado con la asociación de dermatomiositis y colitis ulcerosa. Caso clínico: Paciente de 70 años, femenina, blanca, ama de casa, sin antecedentes familiares de interés ni epidemiológicos. Refiere que a los 50 años presentó cuadros diarreicos acompañados de dolor abdominal, moco y pus. Desde hace 1 año aproximadamente ha estado presentando debilidad muscular a nivel de los hombros, lo que le dificultaba levantar los brazos. Se realizaron exámenes de laboratorio, imagenológicos e histopatológicos que mostraron la presencia de una dermatomiositis asociada con colitis ulcerativa. Se señalan las características clínicas, medios diagnósticos y la terapia empleada en nuestra paciente. Conclusiones: se presentó el caso de una paciente con manifestaciones clínicas de dermatomiositis y colitis ulcerativa. Esta asociación es muy infrecuente y debe ser tenida en cuenta por los médicos que reciben consultas de estos pacientes, debido a que ambas entidades tienen una base inmunológica y pueden expresarse de variadas formas.
\end{abstract}

Palabras clave

Dermatomiositis, colitis ulcerosa.

\begin{abstract}
Introduction: The incidence of idiopathic inflammatory myopathy ranges from 4 to 15 cases per million inhabitants while its prevalence is 60 cases per million inhabitants. Given the identification of clinical forms, it is possible that these figures will increase in the coming years. Incidence and prevalence of ulcerative colitis have been increasing, and it now affects $2 \%$ of the world population. Symptoms are determined by the extent and severity of lesions. It is chronic with recurring outbreaks, and there is no medical cure. Dermatomyositis in association with ulcerative colitis occurs extremely infrequently. Objective: This study presents the case of a patient diagnosed with dermatomyositis associated with ulcerative colitis. Clinical Case: The patient was a 70 years white woman, a housewife with no family or epidemiological history of interest. She reported that she had had diarrhea accompanied by abdominal pain, mucus and pus when she was fifty years old. About one year prior to diagnosis, she developed muscle weakness in her shoulders that made it difficult for her to lift her arms. Laboratory, imaging and histopathological tests showed dermatomyositis associated with ulcerative colitis. We point out clinical characteristics, diagnostic means and the therapy used to treat our patient. Conclusions: This case of a patient with clinical manifestations of the very infrequent combination of dermatomyositis and ulcerative colitis should be taken into account by doctors who care for these patients because both conditions have immunological bases and can be expressed in various ways.
\end{abstract}

\section{Keywords}

Dermatomyositis, ulcerative colitis. 


\section{INTRODUCCIÓN}

La polimiositis fue descrita por Wagner en 1882 y en 1887 Ulverricht denominó dermatomiositis (DM) a la asociación de dolor y debilidad muscular con lesiones cutáneas inflamatorias. Hachulla (1) señaló que los criterios de Bohan y Peter (2) son útiles para diferenciar la patogenia de estas miopatías: pérdida simétrica y progresiva de la fuerza muscular proximal de las cinturas con o sin disfagia, o afectación de los músculos respiratorios; elevación de las enzimas musculoesqueléticas; biopsia muscular; alteraciones electromiográficas y exantema cutáneo.

La DM se presenta de forma secundaria a neoplasias localizadas en el pulmón y tubo digestivo fundamentalmente. Sin embargo, su asociación con la colitis ulcerativa (CU) es muy infrecuente y constituye el primer caso reportado en nuestro hospital en 55 años.

\section{CASO CLÍNICO}

Una paciente de 70 años, femenina, blanca, ama de casa, sin antecedentes familiares de interés ni epidemiológicos refirió que a los 50 años presentó cuadros diarreicos acompañados de dolor abdominal, moco y pus; con una frecuencia diaria entre 6 y 8 deposiciones y una duración entre 5 a 7 días, que mejoraba con dieta. Acudió a consulta en varias ocasiones, sin realizarse el diagnóstico. Todo este cuadro duró aproximadamente 5 años alternando períodos de mejoría clínica con la sintomatología descrita. Desde hace 1 año aproximadamente ha estado presentando debilidad muscular al nivel de los hombros, lo que le dificultaba levantar los brazos, peinarse y realizar labores caseras cotidianas. Además, ha notado dificultad para levantarse de la posición de sentada y no puede subir escalones ni deglutir alimentos sólidos. Todo este cuadro se presentó asociado con lesiones de la piel alrededor de los ojos, tórax y ambas manos.

\section{EXPLORACIÓN CLÍNICA}

Se hallaron mucosas húmedas y coloreadas, y lesiones con aspecto de heliotropo en ambas zonas periorbitarias (Figura 1).

Lesiones eritematosas en diferentes zonas del cuerpo, más acentuadas en la parte anterosuperior del tórax, brazos y muslos (Figura 2).

En el examen físico, la paciente presentó las características descritas a continuación:

- Sistema cardiorrespiratorio: murmullo vesicular normal, tonos cardíacos rítmicos, tensión arterial: 120/80 $\mathrm{mm} \mathrm{Hg}$, frecuencia cardíaca central: 80 latidos por minuto (lpm).
- Abdomen: sin visceromegalia, dolor a la palpación profunda en hemiabdomen izquierdo.

- Sistema nervioso: orientada en tiempo, espacio y persona. Sin signos focales.

- Sistema osteomioarticular: dolores articulares generalizados fundamentalmente en ambas manos; donde se aprecian lesiones papuloeritematosas descamativas situadas en el dorso de las manos y zonas metacarpofalángicas e interfalángicas (signo de Gottron) (Figura 3); acompañadas de mialgias progresivas, pero más severas en la región proximal de ambos brazos; llama la atención la hipotrofia muscular de varios músculos. Los reflejos osteotendinosos estaban disminuidos y la fuerza muscular disminuida en las 4 extremidades.

- Tacto rectal: heces mucosas teñidas de sangre con dolor a la realización del mismo.

- Tacto vaginal, mamas y fondo de ojo sin alteraciones.

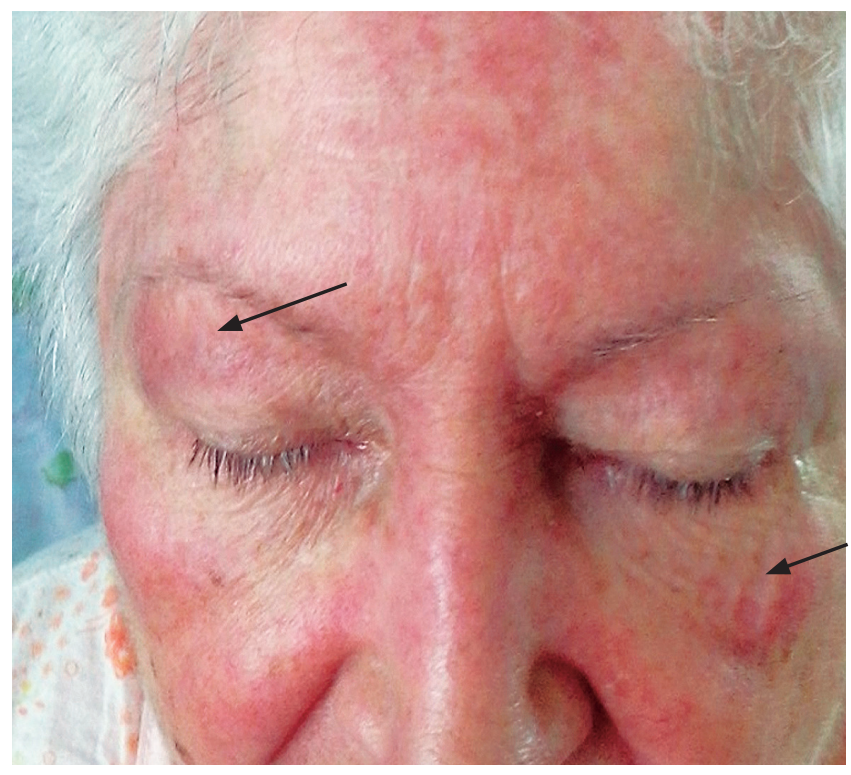

Figura 1. Eritema en heliotropo. Obsérvese la tonalidad violácea de las lesiones y su distribución alrededor de los ojos y los párpados de forma bilateral y simétrica acompañadas de un cierto grado de edema.

\section{ESTUDIO ANALÍTICO}

En el estudio analítico la paciente presentó las características descritas a continuación:

- Hemoglobina: $11 \mathrm{~g} / \mathrm{L}$, leucocitos: 10,3 × 109/L con diferencial normal, velocidad de sedimentación globular (VSG): $70 \mathrm{~mm} /$ primera hora. Glucemia, creatinina, lipidograma, ácido úrico, conteo de Addis y venereal disease research laboratory (VDRL) normales. Proteínas totales: albúmina: $64,5 \% ; \alpha_{1}: 1,3 \% ; \alpha_{2}: 2,5 \% ; \beta: 4,4 \% ; \gamma: 12,6 \%$. Células LE, factor reumatoideo, anticuerpos antinuclea- 


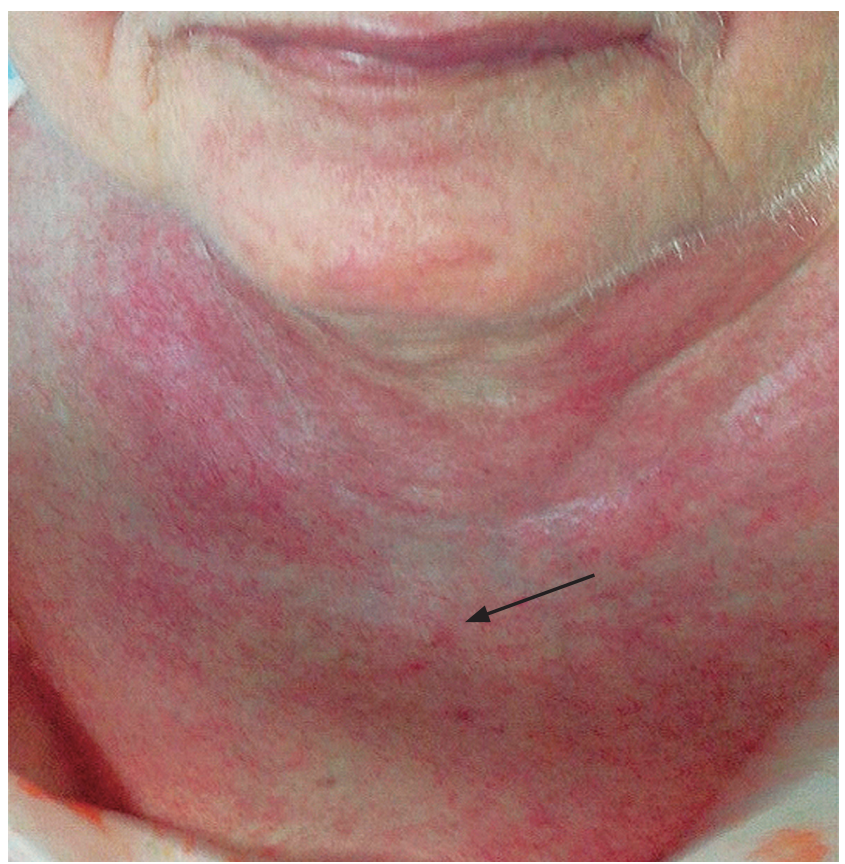

Figura 2. Eritema en la cara anterior del cuello y la $\ll V \gg$ del escote.

res, crioglobulinas, complemento y anticuerpo antifosfolípidos negativos.

- Enzimas: creatina-fosfocinasa (CPK): $1250 \mathrm{UI}$, el isoenzimograma señaló elevación de la MM. Transaminasa glutámica oxalacética (TGO): $70 \mathrm{UI}, y$ transaminasa pirúvica (TGP): 63 UI. Deshidrogenasa láctica (LDH): 580 UI a expensas de las fracciones II y III. Perfil de tiroides normal. Coprocultivo y heces fecales: negativos de bacterias y parásitos. Virus de la inmunodeficiencia humana (VIH), antígenos para hepatitis B, anticuerpos contra hepatitis $\mathrm{C}$, serología lenta en busca de brucela y hemocultivos negativos.

- Radiografía de tórax: normal. Radiografía de ambas manos y ambas articulaciones: disminución de los espacios articulares metacarpofalángicos e interfalángicos con cambios degenerativos generalizados. Ultrasonido abdominal y ginecológico: se aprecia engrosamiento de la capa mucosa del colon izquierdo, atrofia de genitales internos. Electrocardiograma (ECG) normal. Endoscopia del tracto digestivo superior: esófago, estómago y duodeno sin alteraciones. Tomografía axial computarizada (TAC) de tórax, abdomen, retroperitoneo y mediastino: acentuados cambios degenerativos de la columna dorsal y lumbar. Sin alteraciones mediastinales, retroperitoneales y abdominales. Espirometría normal.

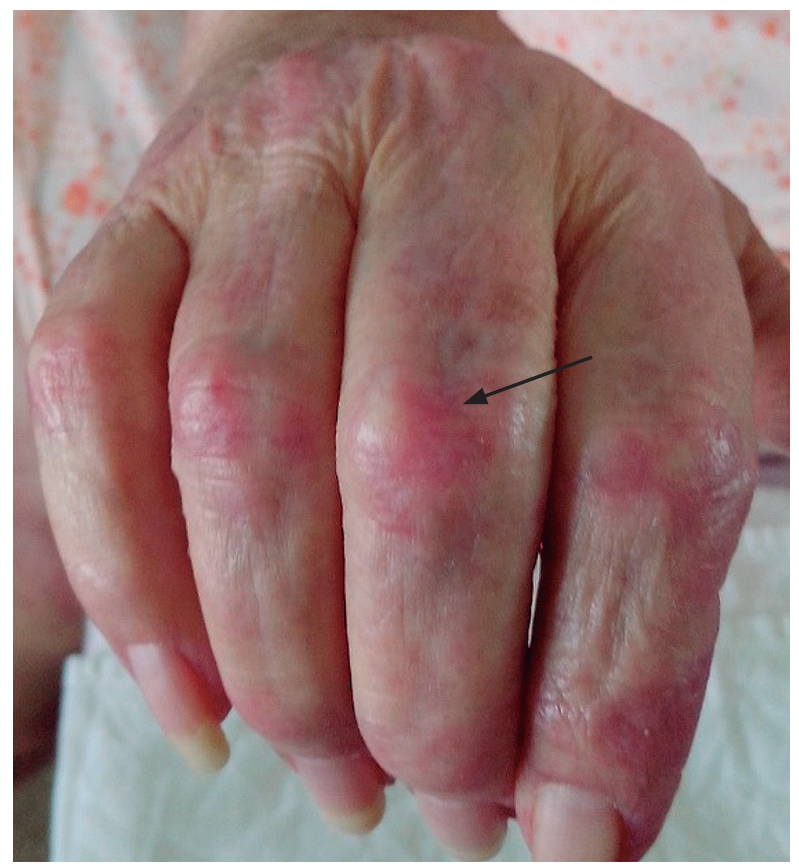

Figura 3. Signo de Gottron.

Electroneurofisiología (ENF) del cubital, mediano y tibial posterior normales. Electromiografía del tibial anterior e interóseos izquierdos presentan fibrilación espontánea que aparece en el reposo. Potencial de unidad motora (PUM) mostró pequeña amplitud y corta duración.

- Biopsia de piel y músculo (Figuras 4 y 5): infiltrado perivascular de linfocitos en el plexo profundo, inflamación a predominio linfocítico de vasos del perimisio y atrofia perifascicular.

Por la posibilidad de tratarse de un cáncer de colon y ser la causa de la DM se realizó una colonoscopia que mostró mucosa eritematosa, friable en una superficie granular con patrón vascular normal. Se tomó una muestra para el estudio histopatológico en el que se encontró linfocitos intraepiteliales difusos dispersos, cuerpos apoptóticos en células epiteliales superficiales, metaplasia de células de Paneth, incremento celular mononuclear en la lámina propia y en el estudio inmunohistoquímico: $\mathrm{CD}_{8}+>25 \%$ de las células. Todos estos hallazgos son compatibles con una $\mathrm{CU}$ (Figura 6 A y B).

Como se pudo llegar con mucha dificultad al colon izquierdo y tomar la biopsia, se practicó un enema opaco (Figura 7) y los hallazgos coincidieron con los reportados por la colonoscopia, negando la posibilidad de una neoplasia. 


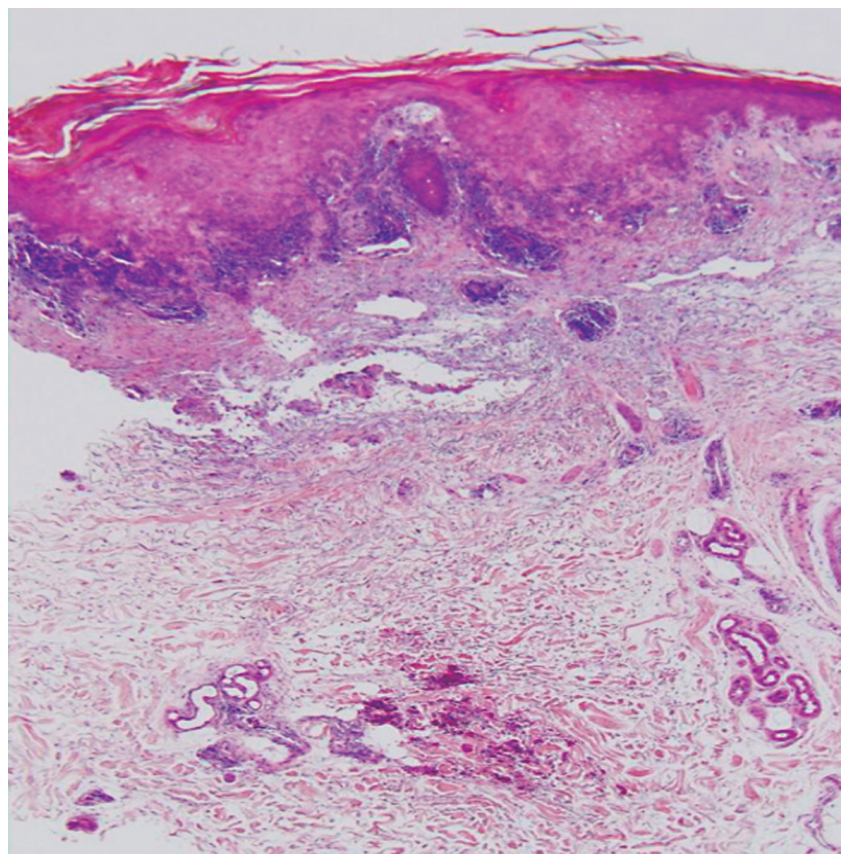

Figura 4. Corte histológico panorámico de piel. Obsérvese el infiltrado inflamatorio perivascular de la dermis papilar y de la dermis profunda (H/E 20x).

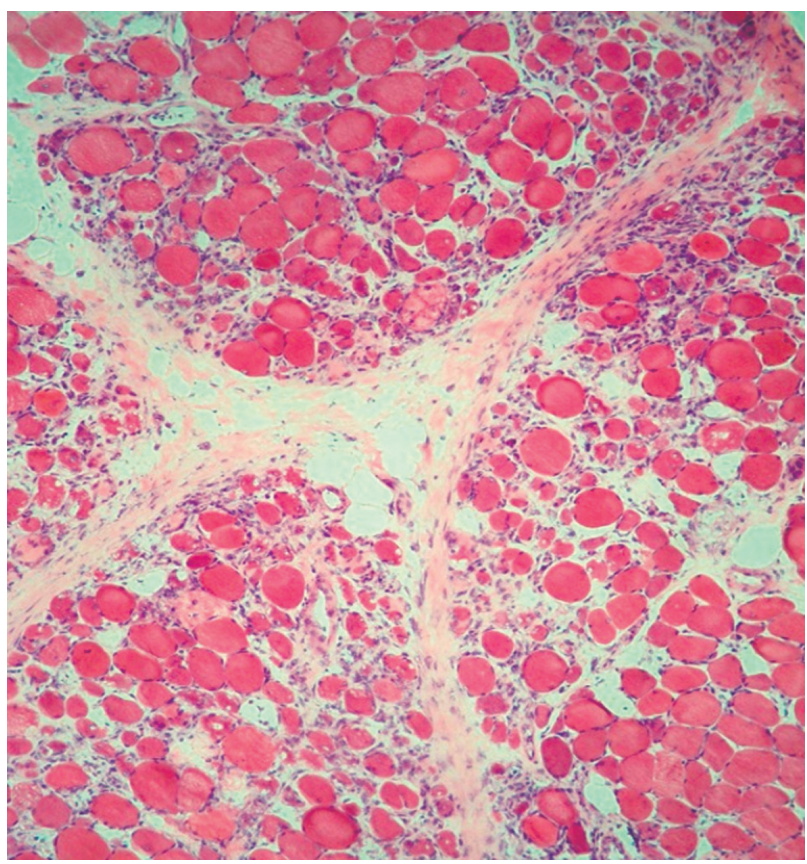

Figura 5. Campo histológico del músculo deltoides que muestra inflamación perimicial y endomicial de predominio linfocítico con necrosis y atrofia de fibras (H/E 40x).
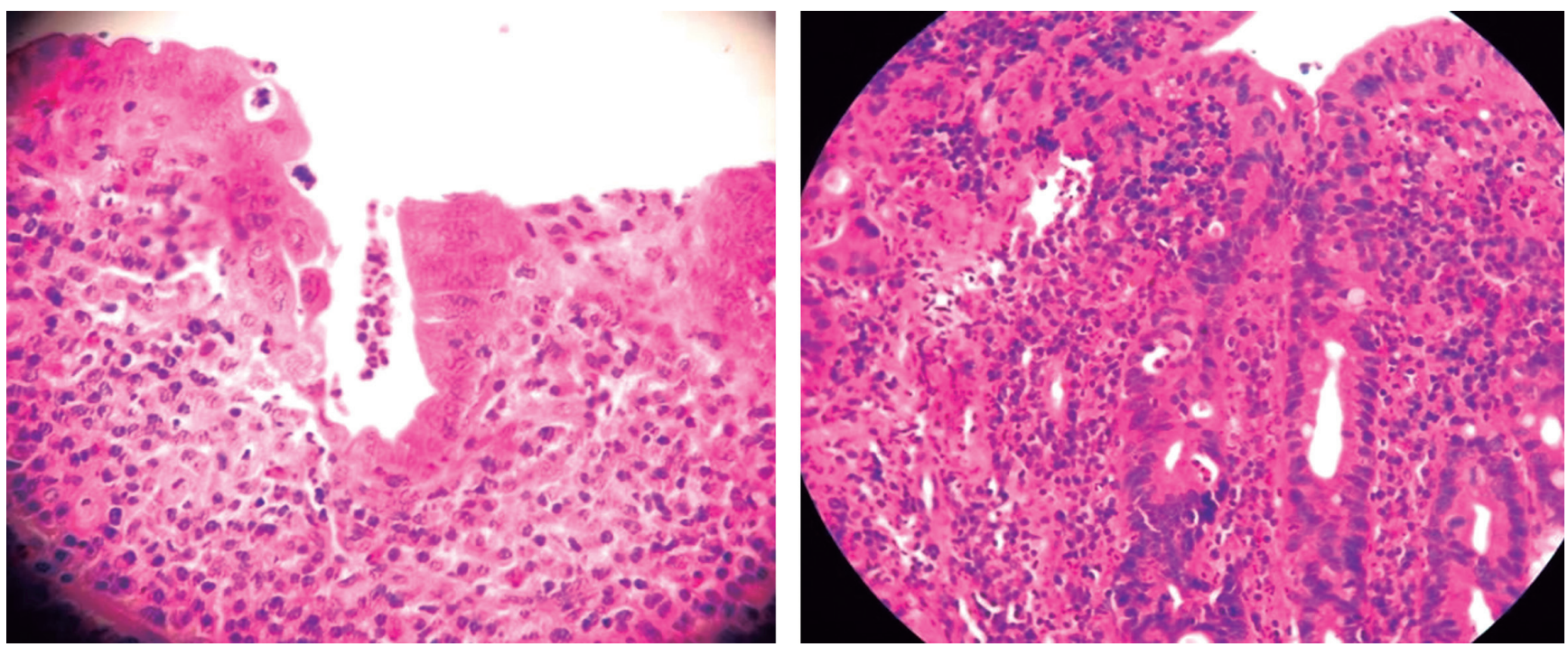

Figura 6. A y B. Microfotografía histológica de la biopsia del colon con presencia de inflamación crónica severa de toda la mucosa y en la que se aprecia criptitis típica de la enfermedad (H/ E 40x).

La paciente recibió tratamiento con prednisona a razón de $1 \mathrm{mg} / \mathrm{kg} /$ día, junto con azulfidina $500 \mathrm{mg}$ cada 6 horas las primeras semanas y después 2 tabletas cada 12 horas al egreso. Este tratamiento fue eficaz ya que la paciente ha presentado una importante mejoría clínica.

\section{DISCUSIÓN}

El diagnóstico de una miopatía inflamatoria debe considerarse cuando los pacientes presentan debilidad de las extremidades superiores e inferiores sin síntomas sensoriales; 


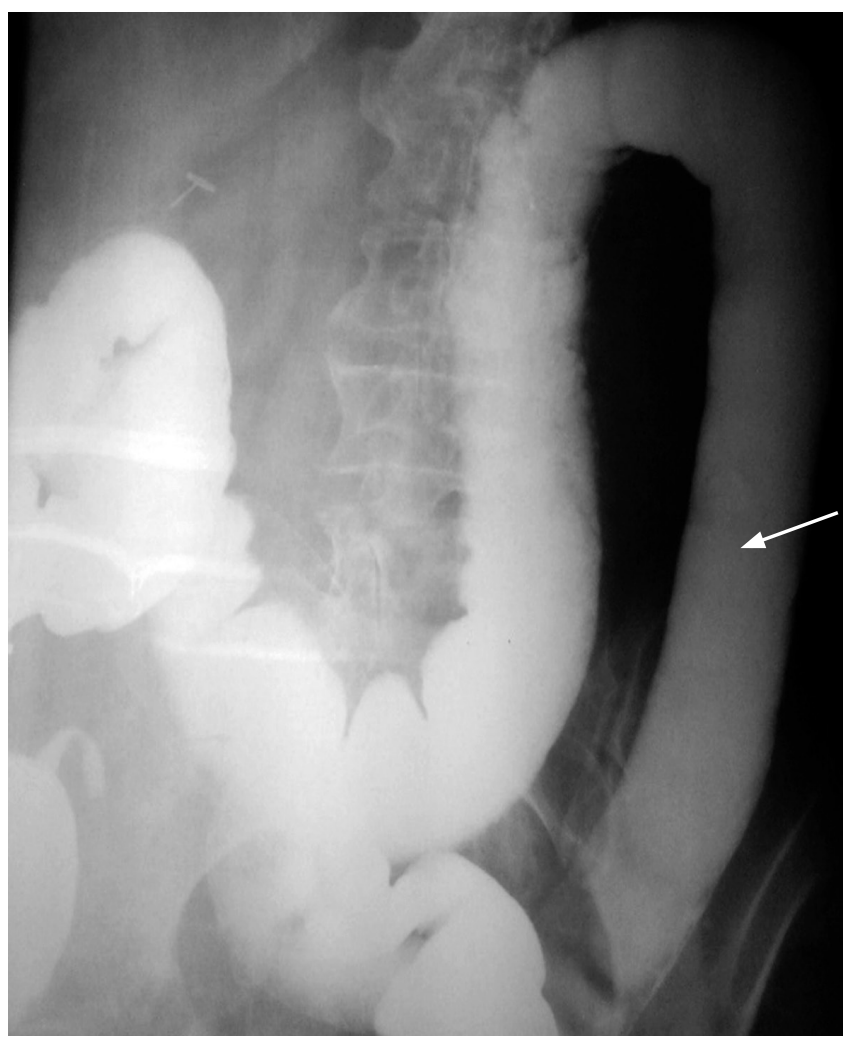

Figura 7. Colon por enema. Obsérvese la pérdida de las haustras con rigidez de la pared del colon descendente y sigmoide.

o con las lesiones típicas características de la DM (1-4). Las lesiones patognomónicas en la piel son el exantema en heliotropo, que es violáceo periorbitario con edema macular. En otras ocasiones se presenta el signo de Gottron, que son pápulas violáceas que aparecen en el dorso de las articulaciones de ambas manos, como se observaron en esta paciente. Igualmente, pueden ocurrir telangiectasias periungueales, trombosis de capilares, poiquilodermia y áreas de fotosensibilidad en zonas de exposición conocidas como el signo del mantón.

Existen otros síntomas y signos que pueden presentarse, como neumonitis intersticial, afectación cardíaca y disfagia, como se observó en esta paciente. Las alteraciones enzimáticas propias de la DM plantean diagnósticos diferenciales como enfermedades malignas, hepatopatías e infarto del miocardio. La DM en asociación con procesos malignos constituye un síndrome paraneoplásico. Algunos autores (5) señalan la incidencia entre un $13 \%-15 \%$ y otros (6) entre el $20 \%-30 \%$. Los datos ofrecidos por la biopsia de piel y músculo muestran una inflamación perimisial y perivascular, y puede ocurrir atrofia perifascicular, lo que ofrece el diagnóstico.
La CU ocurre en la mayoría de los casos entre la tercera y séptima década de la vida (7), esta paciente tenía 70 años. Cabe recordar que aún no se conoce su causa y el mecanismo por el cual se produce el daño hístico (8). El diagnóstico de esta paciente se fundamentó en los criterios de Mendeloff (9): historia de diarreas o sangramientos rectales por 6 semanas o más, sigmoidoscopia que revele friabilidad de la mucosa con sangramiento al contacto o hemorragias petequiales con inflamación de la mucosa, enema de bario con evidencias radiográficas de ulceración, estrechamiento o acintamiento del colon y alteraciones macroscópicas o microscópicas características. Todas estas alteraciones estaban presentes en este caso.

La aparición de complicaciones extracolónicas es un hecho bien conocido, su incidencia oscila entre $25 \%$-36 $\%$ (10). Los órganos afectados con mayor frecuencia son las articulaciones, piel, ojos y sistema hepatobiliar $(7,10$, 11). Como puede apreciarse, la afectación de una DM con una CU es muy infrecuente y no se encontró ningún caso reportado en la literatura médica en el rastreo bibliográfico practicado. Los síntomas musculoesqueléticos son los más frecuentes de esta enfermedad (10); se han descrito artritis similar a la artritis reumatoide, espondilitis anquilopoyética y artritis tóxica aguda (11); en esta paciente solo se reportaron artralgias generalizadas.

En relación con las complicaciones colónicas, se estima que el riesgo de cáncer colorrectal en la CU es de 4 a 20 veces mayor que en la población general (7). Por ello, teniendo en cuenta la posibilidad de esta complicación, se realizaron todos los estudios para descartar la enfermedad, y se diagnosticó la CU por estudios endoscópicos, imagenológicos e histológicos.

Los esteroides son el tratamiento de elección en la DM (12). La prednisona en dosis de $1 \mathrm{mg} / \mathrm{kg} /$ día en dosis inicial de $60 \mathrm{mg}$ es lo que se considera más aconsejable, después de transcurridas 6 a 8 semanas de tratamiento puede iniciarse una reducción lenta de la dosis y el tratamiento debe durar de 12 a 18 meses. Para el tratamiento de la CU se emplearon los aminosalicilatos de la primera generación, la azulfidina de $500 \mathrm{mg}$ en dosis de mantenimiento cada 6 horas y al egreso, 2 tabletas diarias. Con este tratamiento se logró una mejoría notable tanto de clínica (los niveles de enzimas séricas) como de las manifestaciones sistémicas que se presentaban.

\section{CONCLUSIONES}

Se reportó el caso de una paciente con historia previa de síntomas gastrointestinales que acudió a nuestro hospital con manifestaciones articulares, musculares y dermatológicas. En el examen físico y los exámenes complementarios se constató la presencia de una $\mathrm{DM}$ asociada a $\mathrm{CU}$, aunque 
se pensó inicialmente en la posibilidad de una neoplasia de colon. Debe tenerse siempre presente este diagnóstico para realizar un tratamiento oportuno.

\section{REFERENCIAS}

1. Hachulla E. Dermatomyositis and polymyositis: clinical aspects and treatment. Ann Med Interne (Paris). 2001;152(7):455-64.

2. Bohan A, Peter JB. Polymyositis and dermatomyositis (first of two parts). N Engl J Med. 1975;292(7):344-7. doi: 10.1056/NEJM197502132920706.

3. García Gutiérrez A, Porro Novo N, Brito E. Colitis ulcerativa idiopática. En: Pardo Gómez G, García Gutiérrez A (editores). Temas de cirugía. La Habana: Ciencias Médicas; 2011. p. 797-817.

4. Miller F. Polimiositis y dermatomiositis. En: Goldman L, Schafer AI (editores). Cecil y Goldman tratado de medicina interna. 24. ${ }^{\text {a }}$ edición. Madrid: Elsevier; 2013. p. 1720-5.

5. Miller $\mathrm{M}$, Vleugels $\mathrm{R}$. Clinical manifestations of Dermatomyositis and Polymyositis in adult. UpToDate [internet] 2013 [acceso el 5 de febrero de 2018]. Disponible en: https://www.uptodate.com/contents/clinical-manifestations-of-dermatomyositis-and-polymyositis-in-adults.
6. Zahr ZA, Baer AN. Malignancy in myositis. Curr Rheumatol Rep. 2011;13(3):208-15. doi: 10.1007/s11926-011-0169-7.

7. Hano García O, Andrade Gomes S, Villa Jiménez O, González Fabian L, Wood Rodríguez L. Caracterización de pacientes con colitis ulcerosa atendidos en centro de nivel terciario. Rev Cub Med [internet] 2016 [acceso el 20 de enero de 2018] 55(1). Disponible en: http:// scielo.sld.cu/scielo.php?script=sci_arttext\&pid=S003475232016000100005\&lng=es.

8. Kelly S. Ulcerative colitis. BMJ. 2012;344:e2947. doi: 10.1136/bmj.e2947.

9. Mendeloff AI. Algunas características epidemiológicas de la colitis ulcerativa y enteritis regional. Gastroenterology. 1966;51:742.

10. Blumberg RS. Inflammatory bowel disease: Medical considerations. En: Greenberger NJ, Blumberg RS, Burakoff F (editores). Current diagnosis \& treatment. Gastroenterology, hepatology \& endoscopy. Boston: Mc Graw-Hill; 2009. p. 22-33.

11. Panés Díaz J. Enfermedad inflamatoria intestinal. En: Rozman C, Cardellach F (editores). Farreras Rozman. Medicina interna. Madrid: Elsevier; 2016. p. 171-8.

12. Hengstman GJ, van den Hoogen FH, van Engelen BG. Treatment of the inflammatory myopathies: update and practical recommendations. Expert Opin Pharmacother. 2009;10(7):1183-90. doi: 10.1517/14656560902913815. 